\title{
Impact of mutual coupling on performance of MIMO systems based on network theory analysis
}

\author{
Tuanjie Xue ${ }^{1, a}$, Yueheng Li, Ping Huang, Meiyan Ju, Guoping Tan \\ ${ }^{1}$ College of Computer and Information, Hohai University, Nanjing, Jiangsu, 211100, China \\ a email:xuetj1016@163.com
}

Keywords: Mutual Coupling, Network Theory Analysis, Matching Network, Channel Capacity.

\begin{abstract}
In this paper, we investigated the performance of multiple elements array system such as receive power, signal correlation, channel capacity, and etc., in the presence of mutual coupling based on microwave network theory analysis. Combining S-parameter method and network analysis, the performance of MIMO receiver was studied detailedly. Computer simulations were operated for three different matching conditions: characteristic impedance match, self-impedance match and optimal multiport conjugate (MC) match. The results show that the impact of different matching condition on system's performance is quite distinct, that is, the matching network plays an important role on improving the performance of MIMO system.
\end{abstract}

\section{Introduction}

In wireless communication, multiple input multiple output (MIMO) system equipped with multiple antennas at both transmitter and receiver sides can increase the channel capacity greatly[1][2]. Due to this attractive performance improvement, MIMO technique has been considered by many academic researchers, which leads to a great deal of publications.

However, mutual coupling among antennas occurs when multiple antenna elements are placed in a small assembling place, such as in a mobile phone, a compact MIMO base station platform, etc [3]. Such coupling will lead to increasement of correlation of antenna signals [4]-[5]. Correlation of the receiver signals can considerably decrease the capacity of multiple element array (MEA) systems [6]. [7]-[11] have investigated the impact of different matching networks on the correlation of signals and MIMO capacity.

In this paper, an alternative analysis method based on microwave network theory is presented to describe the compact MIMO communication systems. Using the so-called S-parameter representation, we will analyze the performance of the MIMO systems when there is serious mutual coupling among different antennas elements.

\section{Antenna Network Model}

Fig.1 shows complete network structure model of compact MIMO communication system. Here, we regard $\bar{b}_{0}$ as the excitation source of receiver antenna array. From Fig.1 and microwave network theory [12], we can lead to

$$
\begin{aligned}
& \bar{b}_{R}=\bar{b}_{0}+\overline{\bar{S}}_{R R} \bar{a}_{R} \\
& \bar{a}_{R}=\overline{\bar{S}}_{11} \bar{b}_{R}
\end{aligned}
$$

In order to make the power transmitted to the load maximally, a matching network with scattering matrix $\overline{\bar{S}}_{M}$ is placed in the position between the antenna network and load. It is assumed 
that the matching network is lossless, that is, there is $\bar{S}_{M}^{H} \overline{\bar{S}}_{M}=\overline{\bar{I}}$, where $\overline{\bar{I}}$ is the identity matrix, and $(\bullet)^{H}$ denotes the conjugate transpose operator. $\overline{\bar{S}}_{M}$ can be expressed as[3]

$$
\overline{\bar{S}}_{M}=\left[\begin{array}{ll}
\overline{\bar{S}}_{11} & \overline{\bar{S}}_{12} \\
\overline{\bar{S}}_{21} & \overline{\bar{S}}_{22}
\end{array}\right]
$$

We consider the communication systems with two transmit antennas and two receive antennas for simplicity. We assume that the system is terminated with matched load $Z_{0}$. So only the incident wave $\bar{b}_{R}^{\prime}$ is present, and the reflected wave is non-existent. Combining (1) and (2), we can get the expression of incident waves propagating into the matched loads [3]

$$
\bar{b}_{R}^{\prime}=\overline{\bar{S}}_{21}\left(\overline{\bar{I}}-\overline{\bar{S}}_{R R} \overline{\bar{S}}_{11}\right)^{-1} \bar{b}_{0}
$$

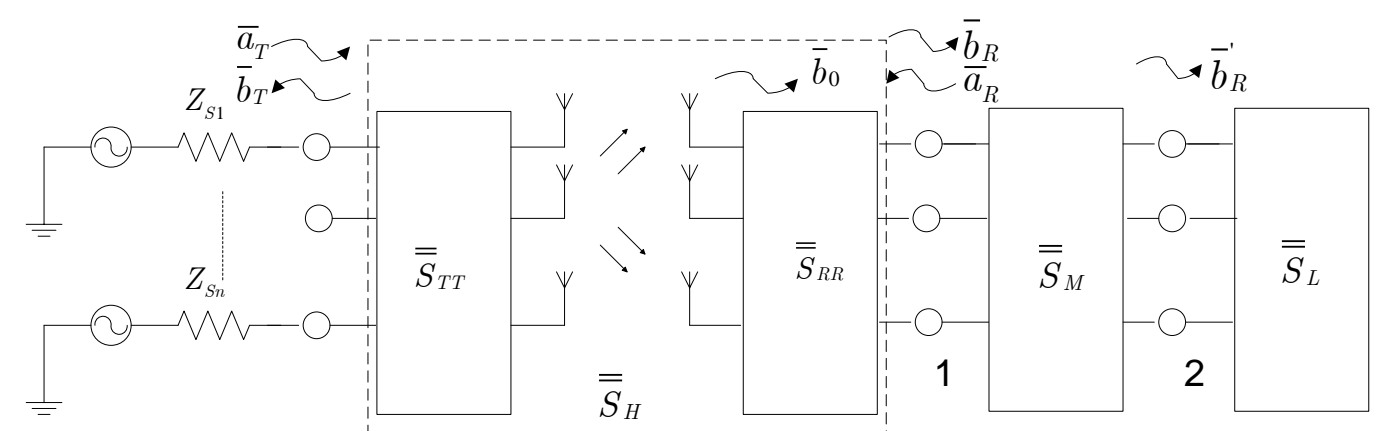

Fig.1 Complete network structure model of compact MIMO communication system

\section{Transmit and Receive Power}

We will derive the close-form expressions for averagely radiated power and receiver power, respectively. According to (4), the total receiver power can be written as

$$
P(\overline{\bar{S}})=\bar{b}_{2}^{H} \bar{b}_{2}=\bar{b}_{0}^{H}\left(\overline{\bar{I}}-\overline{\bar{S}}_{R R} \overline{\bar{S}}_{11}\right)^{(-1) H}\left(\overline{\bar{I}}-\overline{\bar{S}}_{11} \overline{\bar{S}}_{11}\right)\left(\overline{\bar{I}}-\overline{\bar{S}}_{R R} \overline{\bar{S}}_{11}\right)^{-1} \bar{b}_{0}
$$

The averagely collected power is

$$
P_{R}=E\{P(\overline{\bar{S}})\}=\left(\overline{\bar{I}}-\overline{\bar{S}}_{R R} \overline{\bar{S}}_{11}\right)^{(-1) H}\left(\overline{\bar{I}}-\overline{\bar{S}}_{11} \overline{\bar{S}}_{11}\right)\left(\overline{\bar{I}}-\overline{\bar{S}}_{R R} \overline{\bar{S}}_{11}\right)^{-1} \overline{\bar{R}}_{S}
$$

where $\overline{\bar{R}}_{S}=E\left\{\bar{b}_{0} \bar{b}_{0}^{H}\right\}$ and $E\{\bullet\}$ denotes expectation.

For the lossless antennas, the instantaneous radiated power of base station (BS) is

$$
P_{T}^{i n s t}=\left\|\overline{\boldsymbol{a}}_{T}\right\|^{2}-\left\|\bar{b}_{T}\right\|^{2}=\overline{\boldsymbol{a}}_{T}^{H}\left(I-\overline{\bar{S}}_{T T}^{H} \overline{\bar{S}}_{T T}\right) \overline{\boldsymbol{a}}_{T}=\overline{\boldsymbol{a}}_{T} \overline{\bar{A}}_{\overline{\boldsymbol{a}}} \overline{ }_{T}
$$

where $\overline{\bar{A}}=I-\overline{\bar{S}}_{T T}^{H} \overline{\bar{S}}_{T T}$ represents the coupling of transmit antennas.

For zero mean signals, the mean radiated power can be expressed as

$$
P_{T}=E\left\{\bar{a}_{T}^{H} \overline{\bar{A}}_{\bar{a}}\right\}=\operatorname{Tr}\left(\overline{\bar{R}}_{T} \overline{\bar{A}}\right)
$$

where $\overline{\bar{R}}_{T}=E\left\{\overline{\boldsymbol{a}}_{T} \overline{\boldsymbol{a}}_{T}^{H}\right\}$ denotes the covariance of transmit signals. 


\section{Correlation and Channel Capacity}

From Fig.1, the transmit antennas and receive antennas are embedded into scattering medium such that we can write the expression as follows [3]

$$
\left(\begin{array}{l}
\bar{b}_{T} \\
\bar{b}_{R}
\end{array}\right)=\left(\begin{array}{ll}
\overline{\bar{S}}_{T T} & \overline{\bar{S}}_{T R} \\
\overline{\bar{S}}_{R T} & \overline{\bar{S}}_{R R}
\end{array}\right)\left(\begin{array}{l}
\bar{a}_{T} \\
\bar{a}_{R}
\end{array}\right)
$$

Under the condition $\overline{\bar{S}}_{T R}=\overline{\overline{0}}$, the received voltages can be formulated as

$$
\bar{v}_{R}=Z_{0}^{1 / 2} \overline{\bar{S}}_{21}\left(\overline{\bar{I}}-\overline{\bar{S}}_{R R} \overline{\bar{S}}_{11}\right)^{-1} \overline{\bar{S}}_{R T} \bar{a}_{T}
$$

Without loss of generalization, we can assume $Z_{0}=1$, which results in that the covariance of the received voltages can be written as

$$
\overline{\bar{R}}_{L}=E\left\{\bar{v}_{R} \bar{v}_{R}^{H}\right\}=\overline{\bar{S}}_{21}\left(\overline{\bar{I}}-\overline{\bar{S}}_{R R} \overline{\bar{S}}_{11}\right)^{-1} \overline{\bar{R}}_{S}\left(\overline{\bar{I}}-\overline{\bar{S}}_{R R} \overline{\bar{S}}_{11}\right)^{(-1) H} \overline{\bar{S}}_{21}^{H}
$$

For computation of the channel capacity, we assume that the receiver noise model is considered. In this case, yielding the relation as follows

$$
\bar{v}_{R}=Z_{0}^{1 / 2} \overline{\bar{S}}_{21}\left(\overline{\bar{I}}-\overline{\bar{S}}_{R R} \overline{\bar{S}}_{11}\right)^{-1} \overline{\bar{S}}_{R T} \bar{a}_{T}+\bar{N}
$$

where the noise vector $\bar{N}$ is circular symmetric complex Gauss distribution with zero mean and covariance $\overline{\bar{K}}_{N}=\sigma^{2} \overline{\bar{I}}$. Therefore, the mutual information expression is

$$
I(\bar{Y} ; \bar{X})=\log _{2}\left|\frac{\left(\overline{\bar{I}}-\overline{\bar{S}}_{R R} \overline{\bar{S}}_{11}\right)^{(-1) H}\left(\overline{\bar{I}}-\overline{\bar{S}}_{11}^{H} \overline{\bar{S}}_{11}\right)\left(\overline{\bar{I}}-\overline{\bar{S}}_{R R} \overline{\bar{S}}_{11}\right)^{-1} \overline{\bar{S}}_{R T} \overline{\bar{R}}_{T} \overline{\bar{S}}_{R T}^{H}}{\sigma^{2}}+\overline{\bar{I}}\right|
$$

\section{Simulation and Results}

Fig. 2 plots the behavior of the average radiated power, the antenna correlation and the channel capacity over antenna separation. We can see that for the small antenna spacing $(<0.4 \lambda)$, the average transmit power is very low. For $d>0.4 \lambda$, the power tends to be invariant.
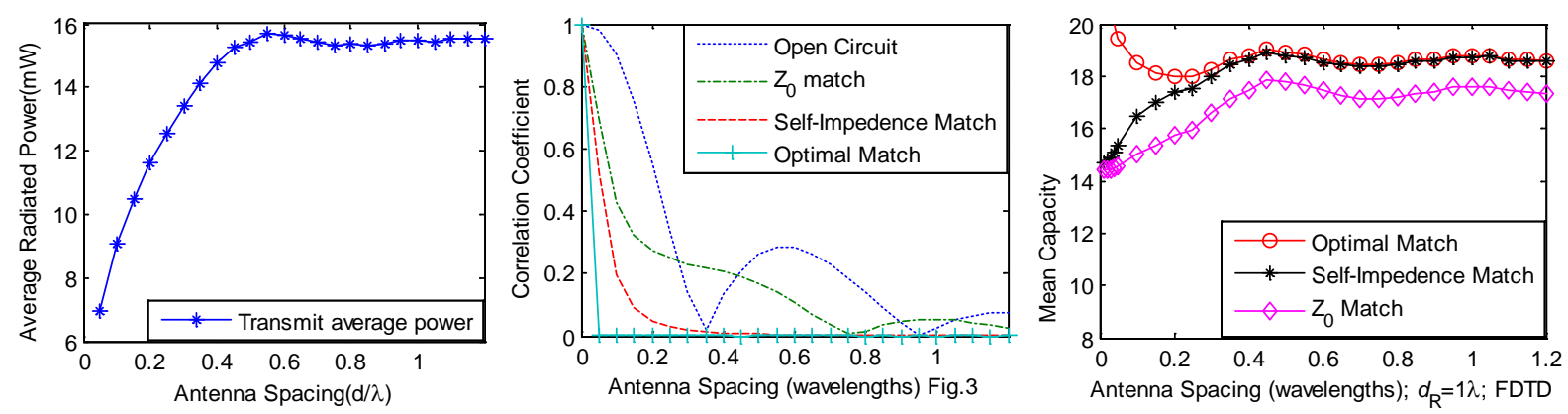

Fig.2 The average radiated power, the antenna correlation and the channel capacity as a function of antenna spacing

We can see from the middle figure, the open circuit termination has the worst performance. MC match has the zero correlation coefficient when the inter-element separation is not zero. We note from the right figure that when the antenna distance is fixed at $1 \lambda$ at the transmitter and for 
$d<0.2 \lambda$, the optimal MC match can obviously get more capacity than other two cases. As the distance among antennas increases, the difference between the MC match and self-impedance match disappears.

\section{Conclusion}

In this paper, we utilized the antenna matching network technique based on microwave network theory to analyze the impact of mutual coupling on the performance of compact MIMO system. Using the proposed network framework, the expressions of both received signals' correlation coefficient and average mutual information were deprived. Simulation results illustrate that the mutual coupling between antennas will degrade the system performance. For close antenna separation, a proper matching network design can effectively decrease the correlation of antenna signal, and then improve remarkably the channel capacity of compact MIMO system.

\section{References}

[1]. G. J. Foschini and M. J. Gans, "On limits of wireless communications in a fading environment when using multiple antennas,” Wireless Personal Communications, vol. 6, pp. 311-335, 1998.

[2]. I. E. Telatar, "Capacity of multi-antenna Gaussian channels," Euro. Trans. Telecommun. vol. 10, pp. 585-595, 1999.

[3]. J. W. Wallace and M. A. Jensen, "Mutual coupling in MIMO wireless systems: a rigorous network theory analysis,” IEEE Trans. Wireless Communication, vol. 3, No 4, pp. 1317-1325, 2004.

[4]. Buon Kiong Lau, Andersen, J.B., Gerhard Kristensson and Andreas F. Molisch , "Impact of Matching Network on Bandwidth of Compact Antenna Arrays,” IEEE Transactions, Antennas and Propagation, vol. 54, pp. 3225-3238, 2006.

[5]. J. W. Wallace and M. A. Jensen, "Impact of Antenna Coupling on Diversity Performance: Complete Network Theory Analysis," in Proceedings of the IEEE International Conference on Communications (ICC '04), vol. 2, pp. 947-951, Paris, France, June 2004.

[6]. D. S. Shiu, G. J. Foschini, M. J. Gan, J. M. Kahn, "Fading correlation and its effect on the capacity of multielement antenna systems ,” IEEE Trans. Commun., vol. 48, pp. 502-513, Mar. 2000.

[7]. Jing Zhang, Hong Bo Zhu and Yan Cai, "Capacity Analysis of Compact MIMO System," 15th Asia-Pacific Conference, 3-7, Shanghai, China, Oct, 2009.

[8]. B. K. Lau, S. M. S. Ow, G. Kristensson, A. F. Molisch "Capacity Analysis for Compact MIMO Systems,” 2005 IEEE 61st Vehicular Technology Conference, 2005. VTC 2005-Spring, vol.1, 165-170, June, 2005.

[9]. J. W. Wallace and M. A. Jensen, "The Capacity of MIMO Wireless Systems with Mutual Coupling," Vehicular Technology Conference, 2002. Proceedings VTC 2002-Fall. IEEE 56th, vol. 2, 2002.

[10]. J. W. Wallace and M. A. Jensen, "Impact of Antenna Coupling on Diversity Performance: Complete Network Theory Analysis," in Proceedings of the IEEE International Conference on Communications (ICC '04), vol. 2, pp. 947-951, Paris, France, June 2004.

[11]. Jianmin Gong, et. al, "The Effect of Antenna Physics on Fading Correlation and the Capacity of Multielement Antenna Systems" IEEE Trans. Commun.vol. 56, no. 4, July. 2007.

[12]. D. M. Pozar, Microwave Engineering, John Wiley \& Sons, 1998. 